Rev.MVZ Córdoba 15(2):2037-2040, 2010.

EDI TORI AL

\title{
¿Ordeñando micobacterias del ganado? I mpacto económico y en salud de Tuberculosis bovina y Paratuberculosis en Colombia
}

\section{¿Milking mycobacteria from cattle? Health and economic impact of bovine Tuberculosis and Paratuberculosis in Colombia}

La tuberculosis bovina y la paratuberculosis o enfermedad de Johne's son enfermedades infecciosas en rumiantes, causadas por $M$. bovis y M. paratuberculosis respectivamente. La tuberculosis bovina es una enfermedad crónica de los rumiantes que guarda estrecha relación con la tuberculosis humana y provoca un deterioro progresivo del estado general de salud del animal, pérdida de peso, muy a menudo con tos y, a la larga, la muerte. La Paratuberculosis es una enfermedad infecto-contagiosa del tracto intestinal y se caracteriza por provocar enteritis granulomatosa crónica y progresiva, y diarreas sin respuesta a un tratamiento. Ambas enfermedades afectan especialmente los bovinos. Ambas bacterias son excretadas en la leche de los bovinos afectados, y por eso el consumo de leche cruda o queso fresco elaborado con leche cruda es la fuente de diseminación más importante de estas enfermedades tanto como entre los animales y al hombre. El impacto de estas enfermedades para la salud humana y la sanidad animal, la economía y el comercio es significativo y por esta razón están entre las enfermedades de declaración obligatoria a La Organización Mundial de Sanidad Animal (OIE) (1).

La Paratuberculosis causa pérdidas económicas en la producción de leche, un aumento de los costos de reemplazo en el rebaño, incremento en la incidencia de mastitis y otras enfermedades infecciosas en el animal infectado y menores ingresos por vaca al momento del sacrificio, contribuyendo a la reducción de los ingresos del ganadero. No hay cálculos sobre el impacto económico de Paratuberculosis en Latinoamérica pero en los EE.UU las pérdidas económicas asociadas con esta enfermedad se han calculado en US\$ 40 por vaca por año cuando el porcentaje de vacas con signos clínicos en el rebaño no es mayor del $10 \%$. Rebaños con más de $20 \%$ de los animales con signos clínicos compatibles con la enfermedad sufren pérdidas económicas de alrededor US\$200 por vaca por año (2). Posiblemente la paratuberculosis también tiene un impacto en la salud humana. Se ha aislado ocasionalmente el agente causal $\mathrm{M}$. paratuberculosis de pacientes con la enfermedad de Crohn, una afección inflamatoria intestinal de los humanos, crónica y dolorosa, con diarreas, que se asemeja a la paratuberculosis bovina. La evidencia actualmente disponible no es suficiente para validar o negar que $\mathrm{M}$. paratuberculosis sea el agente causal de por lo menos algunos casos de enfermedad de Crohn (3). El consumo de leche cruda o queso fresco elaborado con leche no pasteurizada y agua contaminada con la flora intestinal del ganado se consideran como las posibles fuentes de infección.

La razón principal para que la tuberculosis bovina sea una enfermedad de declaración obligatoria es porque puede transmitirse del bovino al humano, especialmente por el consumo de leche o queso crudo, pero también por contacto directo con el animal en la finca o en el matadero. 
En Latinoamérica, en humanos, se registra una baja prevalencia de alrededor de $1 \%$ de la tuberculosis causada por la infección con M. bovis. Sin embargo, se piensa que hay un subregistro y que éste se relaciona con la baja cobertura del cultivo para el diagnóstico de tuberculosis en humanos, especialmente en áreas rurales donde el consumo de leche cruda o queso artesanal es más alto que en la ciudad.

Además la mayoría de los países en Latinoamérica no utilizan un medio suplementado con piruvato, la principal fuente de carbono en el medio de cultivo para M. bovis (4). En países donde la técnicas del laboratorio permiten diferenciar entre una infección por M. bovis o M. tuberculosis, se registran importantes datos casuísticos sobre la infección por $M$. bovis en la tuberculosis humana (5). En Irlanda entre 1998-2006 la infección con M. bovis aportó el 3\% de los casos, en Nueva Zelanda entre 1983-1990 el 7.2\%, en EEUU el 3.0\% entre 19809991 y México en un estudio en 2006-2007, el 13.8\%.

No solamente el impacto en salud humana, también el impacto económico en la productividad de la finca hace importante controlar la tuberculosis bovina. Por ejemplo, un estudio en I rlanda mostró que la producción de leche fue significativamente menor (desde $120 \mathrm{~kg} \mathrm{a}$ $573 \mathrm{~kg}$ de leche por lactancia) para las vacas reactivas a la tuberculina bovina (infectados con M. bovis) en comparación con las vacas no reactivas (6). También la disminución gradual del peso del animal, perdiendo un promedio de $15 \%$ del peso normal y las pérdidas en el momento del sacrificio en el matadero por decomiso parcial o total de los bovinos, afectan la economía de la finca.

La argumentación anteriormente expuesta muestra la importancia de un buen control de ambas enfermedades en nuestros rebaños de producción. Las medidas de prevención y control de la tuberculosis bovina y paratuberculosis comprenden buenas prácticas sanitarias, y el diagnóstico oportuno para identificar los animales infectados. Bovinos infectados deben ser sacrificados en el caso de tuberculosis bovina o ser separados de su cría y los otros animales del rebaño en el caso de paratuberculosis para así proteger los animales y rebaños no infectados. Por tanto, el diagnóstico es crucial en el control de ambas enfermedades.

Para el diagnóstico de tuberculosis bovina y paratuberculosis se utilizan pruebas intradérmicas: la prueba de tuberculina (PPD bovina y PPD aviar). Además, para el diagnóstico de paratuberculosis, existen diferentes ELISAs tanto caseras como comerciales (7). Para el diagnóstico de tuberculosis bovina recientemente fue desarrollada una prueba que mide la respuesta inmunológica del animal a antígenos específicos para la tuberculosis. Esta prueba utiliza sangre completa del animal para determinar la producción de interferón Gamma después el estímulo con los antígenos (8).

Según la información subministrada por el Instituto Colombiano Agropecuario (ICA), en Colombia la prevalencia de tuberculosis bovina es inferior al 1\% y a través de los últimos años, se ha evidenciado la disminución de casos en forma general en el país, a excepción del departamento de Cundinamarca y Boyacá. La Web de esta organización gubernamental además anuncia que se espera para el año 2015, el estatus de país libre de TBC. Sobre la prevalencia de paratuberculosis en Colombia no existe información (9).

Como anteriormente se dijo, paratuberculosis y tuberculosis bovina son enfermedades de declaración obligatoria a la Organización Mundial de Sanidad Animal (OIE), por lo cual los datos que publica esta organización en la Web sirven para evaluar los esfuerzos que hace un país en el control de ambas enfermedades causadas por micobacterias (10).

Colombia, con una población ganadera de aproximadamente 21 millones de cabezas de ganado, reportó entre 2005 y 2009 al OIE 85 brotes de tuberculosis bovina. En este período de 5 años, 7951 animales fueron tuberculinizados (menos que el $0.04 \%$ del ganado colombiano) 
de los cuales 265 (el 3.3\%) resultaron positivos. La campaña de control y erradicación en Colombia aparentemente no es nacional, i.e. no cubre todo el país. El $82 \%$ de los pruebas fueron aplicadas en solamente 4 departamentos (San Andrés 19\%, César 32\% Antioquia $24 \%$ y Cundinamarca $6 \%$ ) olvidándose de los departamentos con mayor densidad de ganado como Córdoba, Sucre y Santander, que tienen más del $20 \%$ de la población bovina del país (10).

Las páginas Web permiten comparar los esfuerzos de Colombia en el control y la erradicación de la tuberculosis bovina con otros países en Latinoamérica. La campaña de erradicación en Argentina, con una población de bovinos de alrededor 51 millones de cabezas, en 2009 aplicó la prueba de tuberculina en 12.955.384 oportunidades detectando 114.170 (1\%) animales positivos. En Brasil en 2008, en un solo año, se reportaron 1.383 brotes con 4.688 animales afectados. También en el país vecino de Venezuela el control de tuberculosis bovina es más activo. En el año 2007, este país reportó a la organización de salud animal la aplicación de 698.451 pruebas de tuberculina detectando 66 brotes con 603 bovinos afectados. Aparentemente en Venezuela, con una población bovina de solamente 12 millones de cabezas, se aplica alrededor de 1000 veces más pruebas que en Colombia en 5 años.

Concerniente a la paratuberculosis, y en contraste con la tuberculosis bovina, Colombia es uno de los pocos países que sí reportó paratuberculosis a la OIE. Información sobre la prevalencia de esta enfermedad en otros países, incluyendo a los exportadores importantes de carne en Latinoamérica como Brasil y Argentina, es nula. Ningún país latinoamericano ha reportado entre el 2005 y el 2009 a la OIE sobre la presencia de paratuberculosis en su territorio. En cambio, en Colombia se reportaron 29 brotes con 3.179 animales ensayados y 50 casos confirmados. Esto es solamente la punta del iceberg teniendo en cuenta que menos del $0,02 \%$ del ganado colombiano fue controlado para esta enfermedad en los últimos 5 años.

Mis propias experiencias con la tuberculosis bovina y paratuberculosis en Colombia son alarmantes. Durante una visita como profesor invitado en junio de este año (2010) a la Facultad de Medicina Veterinaria de la Universidad de Córdoba, en Montería, Colombia, tuve el placer de enseñar a un grupo de estudiantes las técnicas que se utilizan para el diagnóstico y control de ambas enfermedades. En un rebaño de 55 vacas lecheras aplicamos la prueba comparativa con tuberculina PPD aviar y bovina y tomamos muestras de sangre para una ELISA para el diagnóstico de paratuberculosis (Parachek ${ }^{\circledR}$, Prionics AG, Suiza). Además realizamos la prueba de producción de interferón Gamma para el diagnóstico de tuberculosis bovina (Bovigam ${ }^{\circledR}$, Prionics AG, Suiza). En este pequeño estudio piloto se evidenció la presencia de ambas enfermedades en esa finca que fue escogida al azar (finca colaboradora sin intereses de conflicto) y determinamos que el $25 \%$ de este rebaño está infectado con M. paratuberculosis (ELISA y PPD aviar) y dos de los 55 animales resultaron positivos para la infección con M. bovis. (PPD bovino positivo) lo que significa una prevalencia local del $4 \%$. Recordemos que el ICA no ha reportado pruebas de tuberculina en este departamento durante los últimos 5 años.

Estoy consciente que Colombia, por su geografía y difícil acceso a algunas regiones, es un país muy difícil de manejar para controlar las enfermedades endémicas en el ganado. Sin embargo, hay trabajo para hacer. EI ICA debe aumentar las actividades de su programa de vigilancia y llevar su control a todos las provincias del país. Las universidades tienen una tarea importante para iniciar esta investigación. Primordial será la investigación que muestra el impacto de ambas enfermedades tanto en la salud humana como en la economía de la ganadería. Esta línea de investigación es importante para convencer al gobierno y al ganadero que se deben controlar estas enfermedades infecciosas. Además, es importante evaluar pruebas de diagnóstico y desarrollar ELISAs caseros y aplicarlos en estudios de campo para el diagnóstico. También, el seguimiento en el matadero y la confirmación de la infección 
(determinar la especificidad de las pruebas de diagnóstico) son investigaciones necesarias para que el ganadero se convenza que no está sacrificando animales falsos positivos y que puede contar con un rebaño sano y en óptima condición para producir leche y carne de calidad.

Un programa de vigilancia e investigación dirigido a controlar la enfermedad y ofrecer herramientas de diagnóstico debe acabar con la secreción de micobacterias en la leche bovina que afecta a nuestra población y a la productividad de la ganadería. Para expresar mi opinión de una manera más poética: me gustaría terminar con un verso clásico modificado a mi manera de William Shakespeare's Hamlet (escrito en 1600), acto tres, escena uno:

TB or not TB- that is the question:

Whether 'tis nobler in the mind to suffer

The slings and arrows of outrageous fortune,

Or to take arms against a sea of troubles

And, by opposing,

END them

J acobus H. de Waard, Ph.D.

Jefe del Departamento de Tuberculosis, Instituto de Biomedicina, Universidad Central de Venezuela, Caracas. Tel. 58-212-8306670 Correo electrónico: jacobusdeward@gmail.com

\section{REFERENCI AS}

1. Organización Mundial de Salud Animal. Enfermedades de la Lista de la OIE. [en línea].[fecha de acceso agosto de 2010]. URL disponible en: http:// www.oie.int.

2. NAHMS. Johne's Disease on U.S. Dairy Operations. USDA: APHIS:VS, CEAH. Fort Collins, CO: National Animal Health Monitoring System; 1997. \#N245.1097.

3. Calderón Johan, Góngora Agustín. Similaridades clinicopatológicas entre paratuberculosis y enfermedad de Crohn: ¿posible vínculo zoonótico?. Rev MVZ Córdoba 2008; 13(1): 1226-1239.

4. De Kantor I, Ambroggi M, Poggi S, Morcillo $\mathrm{N}$, Da Silva TM, Jacobus $\mathrm{H}$ de Waard, et al. Human Mycobacterium bovis infection in ten Latin American countries. Tuberculosis 2008; 88:358-365.

5. De Waard J. Micobacterias patógenas y la transmisión por alimentos. En: Agentes patógenos transmitidos por alimentos. Volumen II. Ed. Ma. Refugio Torres Vitela y Alejandro Castillo Ayala; 2010. ISBN 978970-764-859-3.
6. Boland F, Kelly GE, Good M, More SJ. Bovine tuberculosis and milk production in infected dairy herds in I reland. Prev Vet Med 2010; 93(2-3): 153-61.

7. Collins MT, Wells SJ, Petrini KR, Collins JE, Schultz RD, Whitlock RH. Evaluation of five antibody detection tests for diagnosis of bovine paratuberculosis. Clin Diagn Lab Immunol 2005; 12(6):685-92.

8. Jacobus H. de Waard. Tuberculosis bovina. In; Manual de Ganaderia doble propósito. Gonzalez-Stagnaro E. Soto-Belloso (eds.). Maracaibo Venezuela: Ediciones Astro Data S.A; 2005.

9. Instituto Colombiano Agropecuario (ICA). [en línea].[fecha de acceso agosto de 2010]. URL disponible en: http:// www.ica.gov.co.

10. Organizacion Mundial de Salud Animal. World Animal Health Information Database (WAHID) [en línea]. [fecha de acceso agosto de 2010]. URL disponible en: http://www.oie.int/ wahis/public. php? page $=$ home. 